\title{
Effect of antidepressant switching between nortriptyline and escitalopram after a failed first antidepressant treatment among patients with major depressive disorder
}

Ole Köhler-Forsberg*, Erik Roj Larsen*, Henriette N. Buttenschøn, Marcella Rietschel, Joanna Hauser, Daniel Souery, Wolfgang Maier, Anne Farmer, Peter McGuffin, Katherine J. Aitchison, Rudolf Uher and Ole Mors

\section{Background}

For patients with major depressive disorder (MDD) experiencing side-effects or non-response to their first antidepressant, little is known regarding the effect of switching between a tricyclic antidepressant (TCA) and a selective serotonin reuptake inhibitor (SSRI).

\section{Aims \\ To compare the switch between the TCA nortriptyline and the SSRI escitalopram.}

\section{Method}

Among 811 adults with MDD treated with nortriptyline or escitalopram for up to 12 weeks, 108 individuals switched from nortriptyline to escitalopram or vice versa because of sideeffects or non-response (trial registration: EudraCT No.2004001723-38 (https://eudract.ema.europa.eu/) and ISRCTN No.03693000 (http://www.controlled-trials.com)). Patients were followed for up to 26 weeks after switching and response was measured with the Montgomery-Åsberg Depression Rating scale (MADRS). We performed adjusted mixed-effects linear regression models with full information maximum likelihood estimation reporting $\beta$-coefficients with 95\% Cls.

\section{Results}

Switching antidepressants resulted in a significant decrease in MADRS scores. This was present for switchers from escitalopram to nortriptyline $(n=36, \beta=-0.38,95 \% \mathrm{Cl}-0.51$ to -0.25 , $P<0.001)$ and from nortriptyline to escitalopram $(n=72, \beta=$ $-0.34,95 \% \mathrm{Cl}-0.41$ to $-0.26, P<0.001)$. Both switching options resulted in significant improvement among individuals who switched because of non-response or side-effects. The results were supported by analyses on other rating scales and symptom dimensions.

\section{Conclusions}

These results suggest that switching from a TCA to an SSRI or vice versa after non-response or side-effects to the first antidepressant may be a viable approach to achieve response among patients with MDD

\section{Declarations of interest}

K.J.A. holds an Alberta Centennial Addiction and Mental Health Research Chair, funded by the Government of Alberta. K.J.A. has been a member of various advisory boards, received consultancy fees and honoraria, and has received research grants from various companies including Johnson and Johnson

Pharmaceuticals Research and Development and Bristol-Myers Squibb Pharmaceuticals Limited. D.S. has served on advisory boards for, and received unrestricted grants from, Lundbeck and AstraZeneca. A.F. and P.M. have received honoraria for participating in expert panels for Lundbeck and GlaxoSmithKline.

\section{Keywords}

Depression; non-responders; side-effects; antidepressants; switching.

\section{Copyright and usage}

(C) The Royal College of Psychiatrists 2019.

\section{Background}

Among patients with major depressive disorder (MDD), guidelines recommend starting antidepressant treatment with a selective serotonin reuptake inhibitor (SSRI), a serotonin norepinephrine reuptake inhibitor (SNRI) or mirtazapine. ${ }^{1}$ Although tricyclic antidepressants (TCA) represent the most efficient group of antidepressants, ${ }^{2,3}$ safety concerns usually reserve initial treatment with a TCA for patients experiencing severe depression. However, despite stateof-the-art treatment, approximately $50 \%$ will not respond sufficiently to their first-line antidepressant. ${ }^{4}$ In order to achieve response, several approaches are relevant. This includes dose increase, ${ }^{5}$ augmentation ${ }^{6}$ or switching to another antidepressant, ${ }^{7}$ with the latter including switching within the same class ${ }^{8}$ or to a different class of antidepressants.

Despite switching between antidepressants representing a frequent and important clinical approach, little research has been performed on this important aspect of clinical decision-making. Recent

\footnotetext{
* These authors contributed equally to this work.
}

meta-analyses found that several studies have investigated switching, but only eight randomised trials have compared the effect of switching antidepressant medication versus continuation., ${ }^{9,10}$ Interestingly, the results showed no difference between switching and continuation. ${ }^{10}$ Indeed, one randomised trial $(n=189)$ found that continuation showed significantly better effects compared with switching. ${ }^{11}$ In addition, the recent VAST-D trial, which included 1522 US veteran patients with MDD and non-response to at least one antidepressant course, ${ }^{12}$ found that augmentation with aripiprazole showed significant better remission compared with individuals randomised to bupropion switching. However, the response rates were rather modest $(22-29 \%)$ and the study population consisted mainly of older males (85.2\%; mean age 54.4 years).

Nevertheless, if continuation is not possible, several switching approaches may improve treatment effects after non-response or side-effects to SSRIs, for example switching to SNRIs ${ }^{5,8}$ or vortioxetine. ${ }^{13}$ Regarding TCA treatment, it is often assumed that the most efficient antidepressant has been given. Therefore, it may not be beneficial to switch to a first-line antidepressant, for example an 
SSRI. Nevertheless, one large trial included patients with chronic depression who failed to respond to a 12-week treatment with the SSRI sertraline or the TCA imipramine. ${ }^{7}$ Switching from sertraline to imipramine $(n=117)$ or imipramine to sertraline $(n=51)$ resulted in response among more than $50 \%$ of the patients in both groups. Another trial found beneficial effects for switching to the SSRI fluoxetine ( $n=142)$ after non-response to the TCA nortriptyline. ${ }^{14}$ However, this randomised study found no difference compared with nortriptyline continuation $(n=68)$.

Hence, more research in this clinically highly relevant area is needed and several switching combinations and specific drugs have not been investigated. ${ }^{10,13,15}$ Furthermore, many patients experience side-effects, particularly to TCA treatment, necessitating switching to another antidepressant. In addition, it has been suggested that switching after 2 weeks may be beneficial among patients with early non-response. ${ }^{16}$ Other findings indicate that continuation and dose increase may lead to better response rates. ${ }^{5}$ The clinically important aspect of switching options after a failed first antidepressant treatment has to be explored in different populations and other SSRI and TCA compounds need to be studied including the effect of the timing of switching. Hence, our aim was to investigate whether switching from the TCA nortriptyline to the SSRI escitalopram or vice versa resulted in improved treatment effects among patients with MDD after a failed first antidepressant treatment.

\section{Method}

\section{Study design and participants}

The GENome-Based Therapeutic Drugs for Depression (GENDEP) study is a 12 -week partly randomised multicentre clinical trial (trial registration: EudraCT No.2004-001723-38 (http://eudract.emea. europa.eu) and ISRCTN No.03693000 (http://www.controlledtrials.com)) comparing treatment with escitalopram with that of nortriptyline (a detailed flow chart is available in Uher et $\mathrm{al}^{17}$ ). A total of 811 adults diagnosed with MDD of at least moderate severity established in the Schedules for Clinical Assessment in Neuropsychiatry (SCAN) interview, ${ }^{18}$ including people both with first-time depression and those with recurrent depression who previously may have received treatment, were recruited in nine European countries. Exclusion criteria were a personal or family history (first-degree relative) of bipolar disorder or schizophrenia, a personal history of hypomanic or manic episodes or mood incongruent psychotic symptoms, active substance dependence, primary organic disease, previous non-response, side-effects or contraindications to both study medications and ethnicity other than White, the latter being because of the genetic part of GENDEP.

We assert that all procedures contributing to this work comply with the ethical standards of the relevant national and institutional committees on human experimentation and with the Helsinki Declaration of 1975, as revised in 2008. All procedures involving human participants/patients were approved by ethics boards in all participating centres. Written informed consent was obtained from all participants. Verbal consent was witnessed and formally recorded.

\section{Interventions}

Participants without contraindications for escitalopram or nortriptyline were randomly allocated to receive one of the two antidepressants using a random number generator, stratified by centre and performed independently of the assessing clinician: 233 were randomised to escitalopram and 235 to nortriptyline. Patients with a history of non-response, side-effects or contraindications for one of the drugs were allocated non-randomly to the other antidepressant: 225 to escitalopram and 118 to nortriptyline.

Escitalopram was initiated at $10 \mathrm{mg}$ daily and increased to a target dose of $15 \mathrm{mg}$ daily within the first 2 weeks and could be further increased to $20 \mathrm{mg}$ daily (and up to $30 \mathrm{mg}$ in individuals where there was clinical agreement that a higher dose was needed). Nortriptyline was initiated at $50 \mathrm{mg}$ daily and titrated to a target dose of $100 \mathrm{mg}$ daily within the first 2 weeks and could be further increased to $150 \mathrm{mg}$ daily (and up to $200 \mathrm{mg}$ individuals where there was clinical agreement that a higher dose was needed). Other psychotropic medications were not allowed with the exception of occasional use of hypnotics. Adherence was monitored weekly by self-reported pill count, and plasma levels of antidepressants were measured at week 8 . Overall, individuals treated with escitalopram or nortriptyline improved to a similar degree regarding depressive symptoms. ${ }^{19}$ If a patient experienced poor tolerance or no response was observed during the first 12 weeks on the initially assigned drug, patients were offered to switch to the other drug. This decision was based on a clinical evaluation considering both therapeutic and adverse effects, as assessed with the antidepressant side-effect checklist (ASEC), ${ }^{20}$ and no cut-off criteria for switching were defined. In total, 72 patients switched from nortriptyline to escitalopram and 36 switched from escitalopram to nortriptyline and were followed for up to 26 weeks after switching. These 108 patients represent the study population for the present study.

\section{Measures}

Depression severity was measured at inclusion and weekly throughout the first 12 weeks, both during the first antidepressant trial and after switching, with a final visit 26 weeks after switching. Three established scales were applied: the clinician-rated 10-item Montgomery-Åsberg Depression Rating Scale (MADRS) ${ }^{21}$ and the 17-item Hamilton Rating Scale for Depression (HDRS), ${ }^{22}$ and the self-report 21-item Beck Depression Inventory (BDI). ${ }^{23}$ We included response, defined as a reduction $\geq 50 \%$ on the MADRS or HDRS and remission, defined as MADRS $\leq 11$ or HDRS $\leq 7$, respectively.

The rating scales were administered by trained psychologists and psychiatrists who achieved high interrater reliability on recorded interviews that did not differ between centres (Cronbach's $\alpha \geq 0.9$ for MADRS and BDI and Cronbach's $\alpha \geq 0.8$ for HDRS). ${ }^{17}$ In a previous GENDEP study ${ }^{19}$ we found that depressive symptoms could be described in more detail by three symptom dimensions derived by categorical item factor analyses: observed mood, cognitive symptoms and neurovegetative symptoms. The observed mood dimension comprises clinician-rated items assessing core mood symptoms, anxiety and activity. The cognitive symptom dimension includes items assessing pessimism, guilt, suicidality and the majority of self-reported items from the BDI. The neurovegetative symptom dimension comprises insomnia, poor appetite, weight loss and decreased libido.

\section{Statistical analysis}

To investigate treatment outcome after switching, we performed mixed-effects linear regression models with full information maximum likelihood estimation and report $\beta$-coefficients including 95\% CIs. These models allow inclusion of all relevant covariates across repeated measurements and efficiently handle missing data. ${ }^{24}$ MADRS was the primary outcome measure as in previous GENDEP studies. ${ }^{17,19}$ Regarding missing data, we found no differences between the switching groups regarding early drop-out before week 12 after switching $(n=40$ dropped out before week 12, $P=$ 0.481 for group difference). Furthermore, we had 26 observations 
with missing single visits on the MADRS and there was a significant difference showing more missing values among those who switched to nortriptyline $(n=16)$ compared with those who switched to escitalopram $(n=10)(P=0.007)$.

First, we performed analyses among all 108 individuals to explore the overall effect of switching. Second, we investigated switchers to escitalopram and switchers to nortriptyline separately on the overall treatment effect and on the treatment effect at every visit during the study period. Third, we performed analyses using the HDRS and the BDI as the dependent variables including analyses on the symptom dimension scores (i.e. the observed mood, cognitive and neurovegetative symptom dimensions). Finally, we explored the effect of switching on response and remission. For this, we performed $t$-tests among all 108 individuals and logistic regression analyses among the 68 individuals who completed 12 weeks of follow-up after switching.

We performed all the above-mentioned analyses in an unadjusted model and in a model adjusting for age, gender, severity of depression at switching and centre. For comparison of demographic and baseline clinical characteristics we performed $t$-tests and report means and standard deviations (s.d.). We used Stata version 14 for all analyses.

\section{Sensitivity analyses}

First, we restricted the analyses to individuals with at least 6 weeks of follow-up after switching to the second antidepressant $(n=84)$. Second, to minimise selection bias, we performed all analyses among individuals who were randomised to treatment (i.e. excluding those 22 patients who had not been randomised). Third, we performed all analyses among individuals with non-response $(n=94)$ to the first medication. This analysis included those individuals who experienced non-response $(n=34)$ and those who experienced both non-response and side-effects $(n=60)$. Fourth, to explore the timing of switching, we investigated whether a specific week of switching was associated with a better response. Fifth, since the two switching groups differed regarding age at onset, we performed analyses where we additionally adjusted for age at onset. Sixth, we performed analyses among those individuals who switched because of side-effects only and among those who switched because of non-response only.

\section{Results}

\section{Participant characteristics}

Characteristics of participants at study entry can be found in Table 1. A total of 72 patients switched from nortriptyline to escitalopram after a mean of 6.2 weeks and 36 from escitalopram to nortriptyline after a mean of 7.0 weeks (range 1-12 weeks for both groups; no difference in switching week, $P=0.197$ ) (Table 2). The reasons for switching were side-effects $(n=12)$, non-response $(n=34)$ or both $(n=60)$, whereas for two individuals there was no information regarding reason for switching (Table 2).

Commonly reported adverse reactions to escitalopram included nausea and vomiting (15\%) and sexual dysfunction (30\%). Common adverse effects of nortriptyline included dry mouth $(80 \%)$, orthostatic dizziness (32\%), drowsiness (27\%) and constipation $(24 \%)$. Individuals who switched from nortriptyline to escitalopram were more often women $(71 \%$ v. $47 \%, P=0.013)$, had a younger age at onset of depressive symptoms (29.5 v. 36.8 years, $P=0.003)$ and were more often randomised to treatment $(86.1 \%$ v. $66.7 \%, P=0.035$ ) (Table 1).

The mean MADRS score at switching was 21.5 (s.d. = 8.3) for those switching from nortriptyline to escitalopram and 24.2 (s.d. = 7.7) for those switching from escitalopram to nortriptyline (Table 3). We found no significant differences in MADRS, HDRS, BDI, observed mood or cognitive symptoms at the time of switching, but those who switched from escitalopram to nortriptyline had higher neurovegetative symptoms ( 0.6 v. $0.1 ; P=0.0009)$ (Table 3 ).

\section{Treatment effect after switching}

Of the 108 individuals, a total of 84 (77.8\%), 81 (75.0\%) and 68 $(63.0 \%)$ completed 6,8 and 12 weeks of follow-up after switching medication, respectively. In addition, 40 (37.0\%) individuals completed the additional follow-up visit 26 weeks after switching. The mean follow-up after switching was 9.5 weeks $($ s.d. $=3.7$ ). We found no difference regarding length of follow-up after switching

Table 1 Characteristics of the study population at study entry (i.e. at the initiation of the first antidepressant trial)

\begin{tabular}{|c|c|c|c|c|}
\hline & Total $(n=108)$ & Nortriptyline $\rightarrow$ escitalopram $(n=72)$ & Escitalopram $\rightarrow$ nortriptyline $(n=36)$ & $P$ \\
\hline Woman, $n$ (\%) & $68(63)$ & $51(70.8)$ & $17(47.2)$ & 0.013 \\
\hline Age, years: mean (s.d.) & $43.1(11.5)$ & $42.0(11.7)$ & $45.2(10.8)$ & 0.164 \\
\hline Randomised, $n(\%)$ & $86(79.6)$ & $62(86.1)$ & $24(66.7)$ & 0.035 \\
\hline Education, years: mean (s.d.) & $12.0(3.1)$ & $12.0(3.3)$ & $11.9(2.7)$ & 0.927 \\
\hline Occupation, $n$ (\%) & & & & 0.878 \\
\hline Full-time work & $37(34.3)$ & $26(36.1)$ & $11(30.6)$ & \\
\hline Part-time work & $13(12.0)$ & 8 (11.1) & $5(13.9)$ & \\
\hline Student & $5(4.6)$ & $4(5.6)$ & $1(2.8)$ & \\
\hline Homemaker & $5(4.6)$ & $3(4.2)$ & $2(5.6)$ & \\
\hline Retired & $16(14.8)$ & $11(15.3)$ & 5 (13.9) & \\
\hline Unemployed & $32(29.6)$ & $20(27.8)$ & $12(33.3)$ & \\
\hline Marital status, $n(\%)$ & & & & 0.405 \\
\hline Married/cohabitant & $57(52.8)$ & $35(48.6)$ & $22(61.1)$ & \\
\hline Separated/divorced & $17(15.7)$ & $13(18.1)$ & $4(11.1)$ & \\
\hline Widowed & $4(3.7)$ & $3(4.2)$ & $1(2.8)$ & \\
\hline Single & $30(27.8)$ & $21(29.2)$ & $9(25.0)$ & \\
\hline Age at onset, years: mean (s.d.) & $31.9(11.9)$ & $29.5(11.4)$ & $36.8(11.6)$ & 0.003 \\
\hline Episodes, $n(\%)$ & & & & 0.392 \\
\hline 1 & 35 (32.4) & $23(31.9)$ & $12(33.3)$ & \\
\hline 2 & $53(49.1)$ & $34(47.2)$ & 19 (52.8) & \\
\hline $3+$ & $20(18.5)$ & $15(20.8)$ & 5 (13.9) & \\
\hline Mean (s.d.) & $1.86(0.70)$ & $1.89(0.72)$ & $1.81(0.67)$ & \\
\hline Current episode duration, weeks: mean (s.d.) & $22.4(17.3)$ & $21.5(16.8)$ & $24.2(18.4)$ & 0.488 \\
\hline
\end{tabular}


Table 2 Clinical characteristics of individuals who switched from nortriptyline to escitalopram $(n=72)$ or escitalopram to nortriptyline $(n=36)$ after nonresponse or side-effects to the first medication

\begin{tabular}{|c|c|c|c|}
\hline & Nortriptyline $\rightarrow$ escitalopram $(n=72)$ & Escitalopram $\rightarrow$ nortriptyline $(n=36)$ & $P$ \\
\hline \multicolumn{4}{|l|}{ Dose before switch, mg: mean (s.d.) } \\
\hline Nortriptyline & $109.3(34.8)$ & & \\
\hline Escitalopram & & $19.6(7.6)$ & \\
\hline Switch < week $8, n(\%)$ & $40(55.6)$ & $16(44.4)$ & 0.325 \\
\hline Switching week, mean (s.d.) & $6.2(2.8)$ & $7.0(3.3)$ & 0.197 \\
\hline Weeks follow-up after switching, mean (s.d.) & $10.0(3.2)$ & $8.7(4.4)$ & 0.099 \\
\hline \multicolumn{4}{|l|}{ Follow-up after switching, $n$ (\%) } \\
\hline$\geq 6$ weeks & $59(81.9)$ & $25(69.4)$ & 0.141 \\
\hline 12 weeks & $47(65.3)$ & $21(58.3)$ & 0.481 \\
\hline Switching reason, $n$ (\%) & & & 0.079 \\
\hline Side-effects & $10(13.9)$ & $2(5.5)$ & \\
\hline Non-response & $18(25.0)$ & $16(44.4)$ & \\
\hline Both & $43(59.7)$ & $17(47.2)$ & \\
\hline Missing information & $1(1.4)$ & $1(2.8)$ & \\
\hline \multicolumn{4}{|c|}{ Weeks follow-up based on reason for switching, mean (s.d.) } \\
\hline Side-effects & $10.1(3.1)$ & $6.5(7.8)$ & 0.323 \\
\hline Non-response & $8.8(3.6)$ & $9.5(3.9)$ & 0.391 \\
\hline Both & $10.4(3.0)$ & $8.0(4.6)$ & 0.186 \\
\hline
\end{tabular}

or reason for switching between the two switching groups (Table 2). Similar proportions of individuals who switched because of nonresponse, side-effects or both completed 6 weeks $(P=0.458)$ or 12 weeks $(P=0.559)$ of the second course of treatment, respectively.

No significant difference was found at the end point between the two switching groups on the three rating scales or the three symptom dimensions (Table 3). The development of symptom scores on the MADRS, HDRS, BDI and the three symptom dimensions before and after switching is illustrated in Fig. 1. Overall, switching resulted in a significant decrease on the MADRS among all 108 patients as indicated by an adjusted $\beta$ of -0.35 (95\% CI -0.41 to $-0.28, P<0.001)$. Both groups improved similarly on the MADRS (escitalopram to nortriptyline: $n=36, \beta=-0.38,95 \%$ CI -0.51 to $-0.25, P<0.001$; nortriptyline to escitalopram: $n=72, \beta=-0.34$, $95 \% \mathrm{CI}-0.41$ to $-0.26, P<0.001)$. We found significant improvements on the HDRS and BDI for both switching groups (Table 3 and Fig. 1 , all $P<0.001)$. Furthermore, both switching groups significantly improved on the three symptom dimensions, i.e. on observed mood, cognitive and neurovegetative symptoms (Fig. 2, all $P<0.001)$. All analyses were adjusted for age, gender, severity of depression at switching, and centre.

\section{Response and remission}

During the entire follow-up period after switching, a total of 58 (53.7\%) individuals achieved responses on the MADRS and 61 (56.5\%) on the HDRS, respectively, whereas 64 (59.3\%) achieved remission on the MADRS and 59 (54.6\%) on the HDRS, respectively. We found no significant differences between the two switching groups regarding response and remission based on $t$-tests (Table 3 ).

When analysing the 68 individuals who completed 12 weeks of follow-up after switching (47 switched to escitalopram and 21 switched to nortriptyline), a total of 28 (59.6\%) switchers to escitalopram experienced response on the MADRS and 22 (46.8\%) experienced remission. Among individuals who switched to nortriptyline, 7 (33.3\%) experienced response and 8 (38.1\%) remission. Fully adjusted logistic regression showed no difference between the two switching groups regarding response $(P=0.125)$ or remission $(P=0.440)$ after 12 weeks, respectively.

\section{Sensitivity analyses}

When only analysing individuals with at least 6 weeks of follow-up after switching, we found significant treatment effects on the
MADRS scale among switchers from nortriptyline to escitalopram ( $n=59, \beta=-0.34,95 \%$ CI -0.41 to $-0.26, P<0.001)$ and switchers from escitalopram to nortriptyline $(n=25, \beta=-0.35,95 \% \mathrm{CI}-0.49$ to $-0.22, P<0.001)$.

Second, when restricting to individuals who were randomised to treatment, we found significant treatment effects on the MADRS among switchers from nortriptyline to escitalopram $(n=62, \beta=$ $-0.34,95 \%$ CI -0.42 to $-0.26, P<0.001)$ and switchers from escitalopram to nortriptyline $(n=24, \beta=-0.41,95 \%$ CI -0.58 to -0.24 , $P<0.001)$.

Third, we found effects for switching among those 94 individuals who switched due to non-response, which was present among individuals who switched from nortriptyline to escitalopram $(n=61, \beta=-0.39,95 \%$ CI -0.47 to $-0.31, P<0.001)$ and individuals who switched from escitalopram to nortriptyline $(n=33$, $\beta=-0.37,95 \%$ CI -0.49 to $-0.25, P<0.001$ ).

Fourth, we performed analyses based on the week of switching (Supplementary Table 1 available at https://doi.org/10.1192/bjp. 2018.302). We found no indication that a specific period, for example early or late switching, showed a better response pattern. Switching in all weeks was associated with better response except for the five individuals who switched in week 4 .

Fifth, analyses additionally adjusting for age at onset supported the primary results. Switchers to escitalopram improved by $\beta=-0.38$ (95\% CI -0.51 to $-0.25, P<0.001$ ), whereas switchers to nortriptyline improved by $\beta=-0.34$ ( $95 \%$ CI -0.41 to $-0.26, P<0.001$ ).

Sixth, analyses among those individuals who switched because of side-effects only $(n=12)$ showed improved treatment effects in both groups (nortriptyline to escitalopram, $n=2, \beta=-1.03$, 95\% CI -1.87 to $-0.19, P=0.016$; escitalopram to nortriptyline, $n=$ $10, \beta=-0.34,95 \%$ CI -0.50 to $-0.17, P<0.001)$, and $9(75 \%)$ out of the 12 achieved remission. The 34 individuals who switched because of non-response only showed improved treatment effects in both groups (nortriptyline to escitalopram, $n=18, \beta=-25$, $95 \%$ CI -0.35 to $-0.15, P<0.001$; escitalopram to nortriptyline, $n=16, \beta=-0.38,95 \%$ CI -0.47 to $-0.29, P<0.001)$ and $17(50 \%)$ out of the 34 achieved remission.

\section{Discussion}

Among 108 individuals with MDD who experienced non-response or side-effects to a first treatment course with escitalopram or 


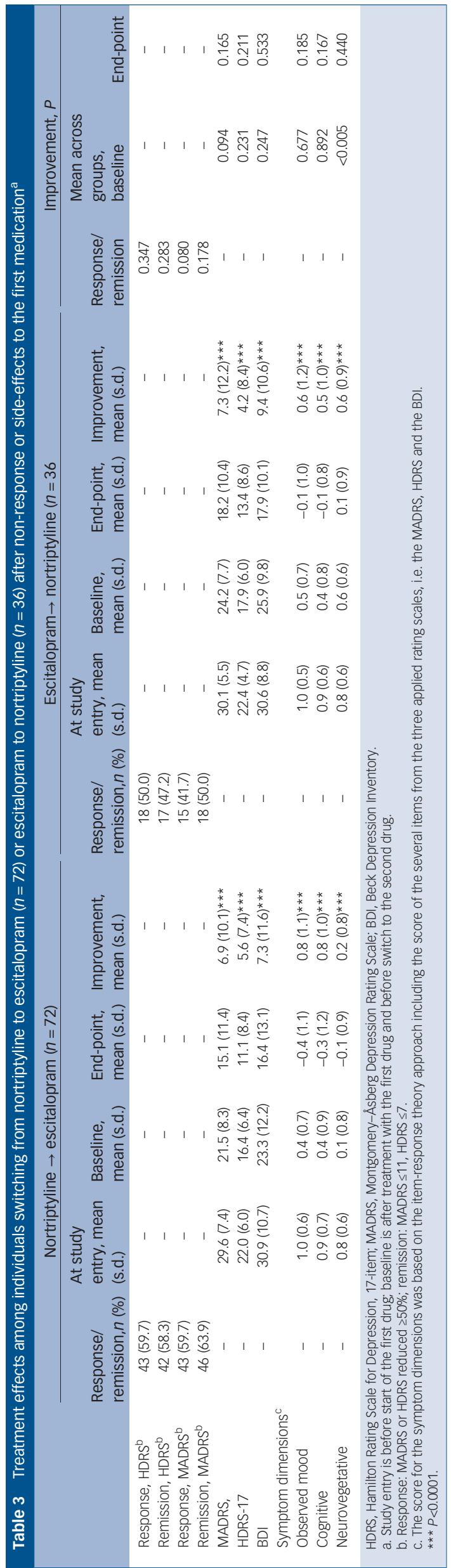

nortriptyline, switching to the other drug resulted in significant improvement in depression scores and more than $50 \%$ of patients achieving response or remission. We found significant improved treatment effects on several rating scales and different symptom dimensions. Particularly, our finding that switching from a TCA to an SSRI improved treatment outcomes is contra intuitive to clinical consensus but in line with two previous trials. ${ }^{7,14}$ However, we had no control group, for example individuals randomised to continuation treatment. Nevertheless, our results suggest that switching from nortriptyline to escitalopram or vice versa may be a viable approach to improve treatment outcomes after non-response or side-effects to a first antidepressant treatment course. Our finding that individuals who switched to escitalopram were younger may be explained by the fact that the first treatment assignment was based on a clinical evaluation, and the rate of contraindications for TCAs increases with age.

\section{Evidence for switching between antidepressants}

Clinicians frequently encounter the situation where continuation with an antidepressant is not possible because of non-response or side-effects. It is important to choose an evidence-based switching strategy with the potential of improving treatment effects. Switching to an antidepressant targeting the same receptors, for example from one SSRI to another SSRI, may seem less likely to result in fewer side-effects and better treatment response. The GENDEP study was designed to investigate two drugs with different mechanisms of action (i.e. escitalopram targeting mainly serotonergic pathways versus nortriptyline mainly targeting noradrenergic pathways). Hence, the important finding of the present study is that switching to an antidepressant with a different receptor profile can improve the antidepressant treatment effects. This is supported by a meta-analysis of clinical trials. ${ }^{25}$ Although only including four trials, the results indicated that after SSRI non-response, switching to a different class of antidepressants showed better response rates compared with switching to a different SSRI. In addition, two prior trials found beneficial effects after switching between a TCA and an SSRI. ${ }^{7,14}$ However, the only randomised trial found no differences between switching to the SSRI fluoxetine $(n=142)$ and nortriptyline continuation $(n=68) .{ }^{14}$

Finally, the timing of switching represents an important clinical aspect. A small study found that among early non-responders to sertraline ( $\leq 20 \%$ reduction in MADRS within the first 2 weeks), randomisation to paroxetine switching $(n=20)$ after 2 weeks resulted in better response and remission compared with sertraline continuation $(n=21) .{ }^{16}$ On the other hand, a larger randomised trial found that after non-response to 2 weeks on escitalopram, continuation and up-titration $(n=229)$ resulted in better response compared with duloxetine switching $(n=245) .5$ In the present study, we could not identify any timing of switching that indicated a better response; however, we were not able to investigate specific hypotheses as the before mentioned studies. Hence, present evidence supports following guidelines before considering switching. Guidelines recommend antidepressant continuation for at least 4-6 weeks to be able to evaluate clinical response. ${ }^{1}$ Indeed, continuation and up-titration can lead to response as late as after 8 weeks. $^{26}$

Future trials may investigate mechanisms potentially explaining better response after switching to specific antidepressant drugs and whether an earlier time of switching may result in better treatment response. Particularly the finding that switching from a TCA to an SSRI improved treatment outcome was unexpected and may be explained by many factors, for example hepatic metabolism or effects on the immune system. 
(a)

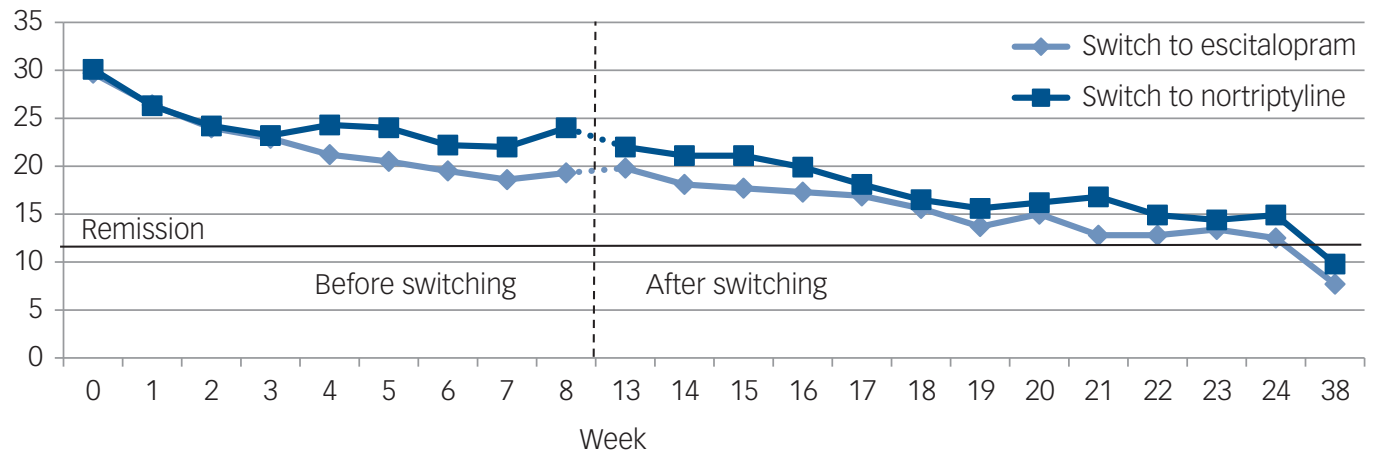

(b)

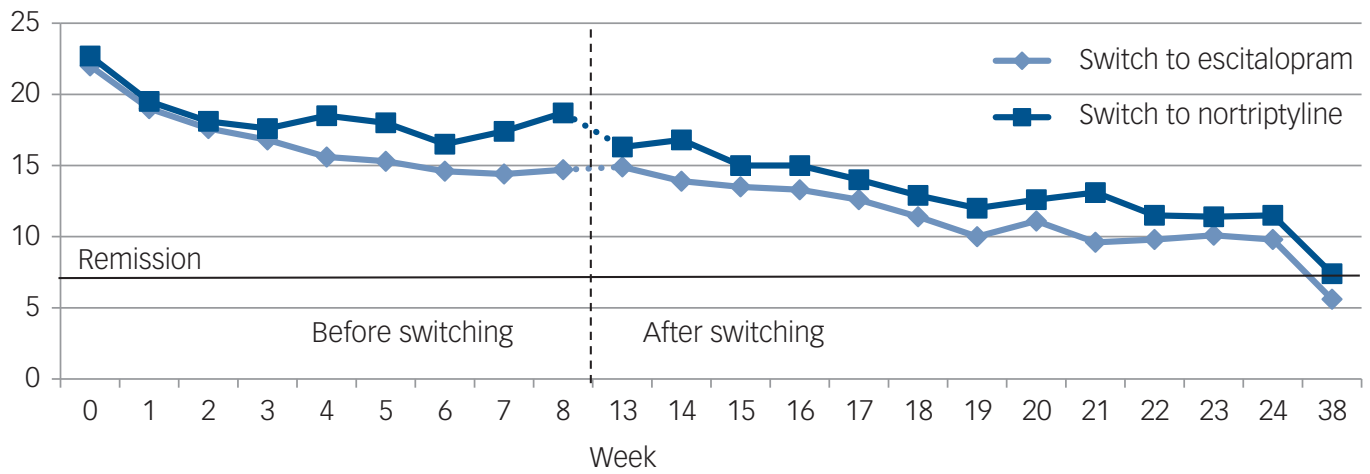

(c)

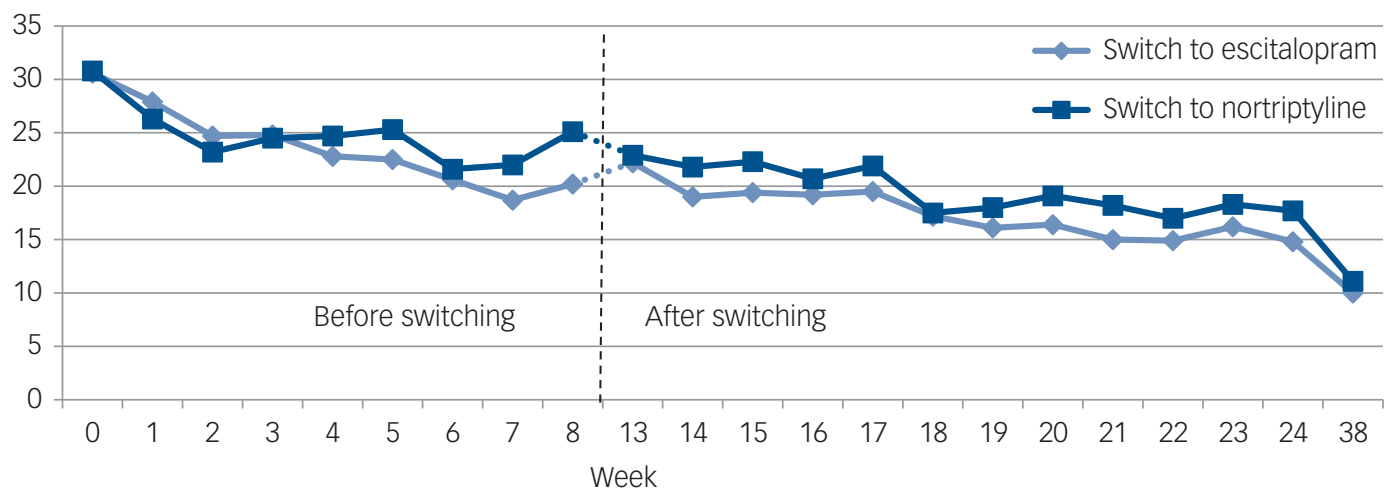

Fig. 1 The development of mean scores on three standard rating scales (a-c) among patients with major depressive disorder (MDD) before (week 0-8) and after (week 13-38) switching from nortriptyline to escitalopram ( $n=72)$ or from escitalopram to nortriptyline $(n=36)$.

Scores on the (a) Montgomery-Åsberg Depression Rating Scale; (b) Hamilton Rating Scale for Depression; and (c) the Beck Depression Inventory. Weeks 9-12 before switching are not shown because of too few individuals.

\section{Strengths and limitations}

GENDEP is a large, real-world partly randomised trial, representing patients seen in everyday practice, that is, with comorbid disorders, prior (failed) antidepressant treatment attempts and suicidal ideation. The close monitoring with weekly visits during 12 weeks of treatment and the large study population (only slightly smaller compared with previous trials that investigated switching between TCAs and SSRIs ${ }^{7,14}$ ) support our findings. Furthermore, the investigated drugs, escitalopram and nortriptyline, target different receptors, which is an important clinical aspect to consider when switching medication.

Regarding limitations, we did not compare the effect of switching with patients randomised to continuation treatment or other approaches such as augmentation. Hence, we are not able to distinguish specific drug response from spontaneous remission and we cannot evaluate whether other treatment approaches, such as augmentation, may have resulted in better treatment response. Furthermore, patients switched at very different time points based on clinical evaluation. This limited the possibility to investigate whether switching at specific time points may result in better treatment effects. In addition, adherence was high (98.4\%) but primarily measured via self-reported pill counts.

\section{Implications}

In the case of a patient with side-effects or non-response despite sufficient dose and treatment duration, switching to an antidepressant with a different receptor profile may be one strategy to improve antidepressant treatment response. Our findings indicate that even switching from a TCA to an SSRI may improve the 
(a)

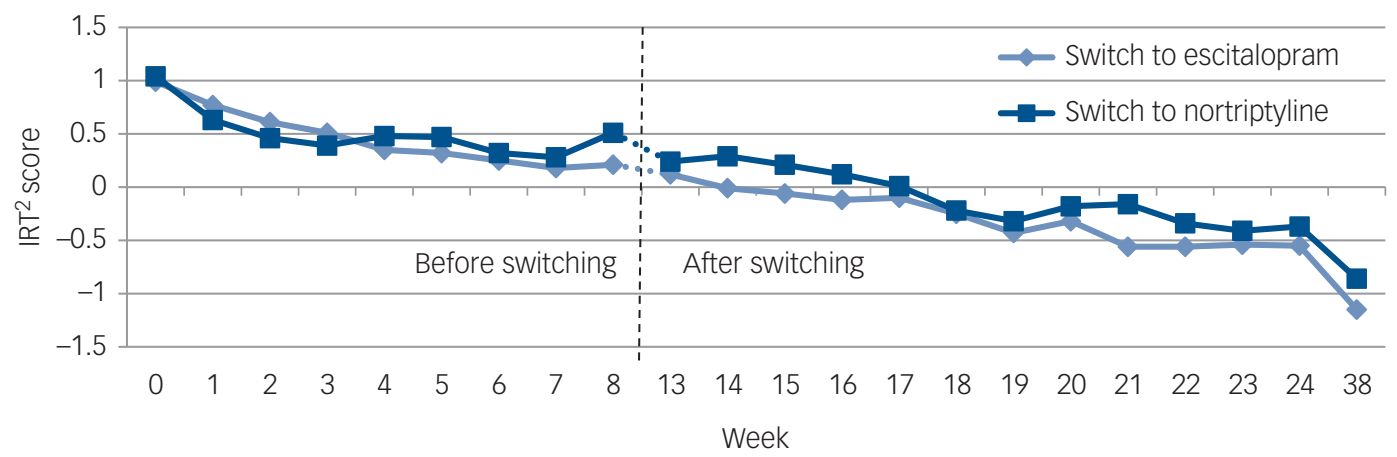

(b)

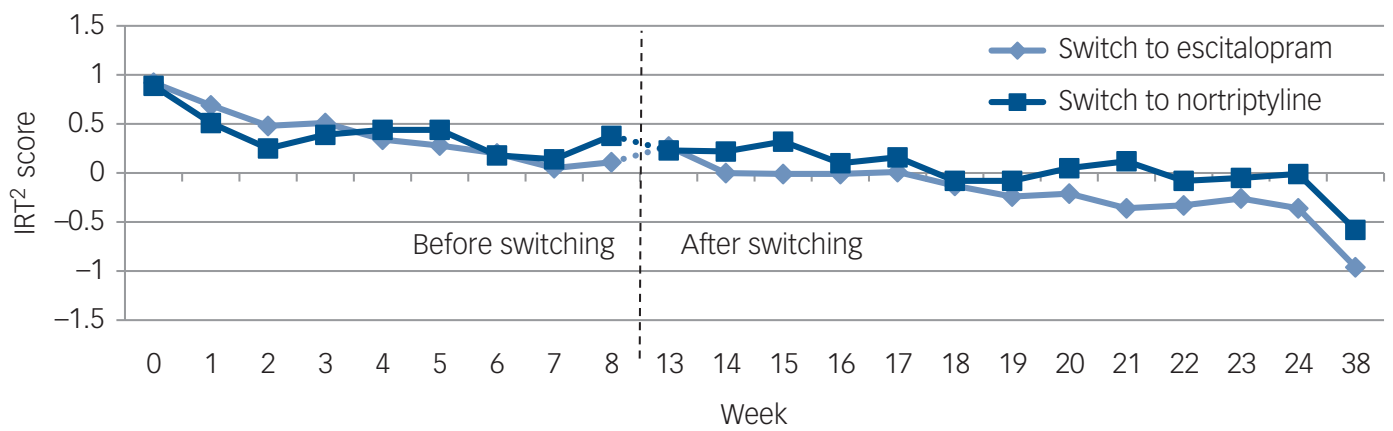

(c)

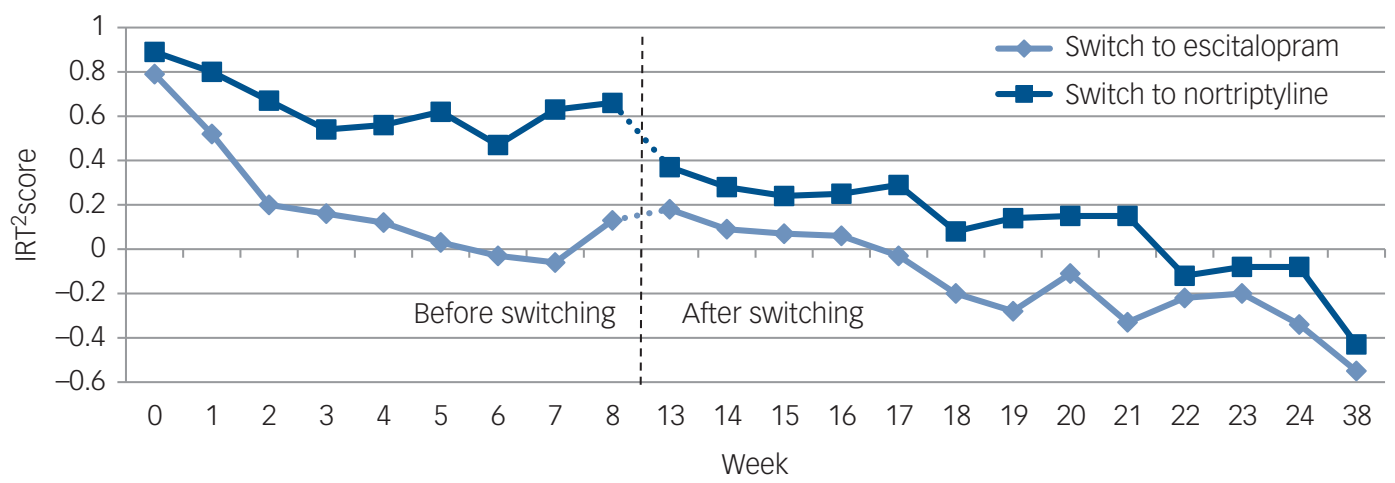

Fig. 2 The development of mean scores on three symptom dimension scores $(a-c)$ among patients with major depressive disorder (MDD) before (week 0-8) and after (week 13-38) switching from nortriptyline to escitalopram ( $n=72)$ or from escitalopram to nortriptyline $(n=36)$.

Scores on the (a) the observed mood dimension; (b) the cognitive symptom dimension; and (c) the neurovegetative symptom dimension. These scores are based on the itemresponse theory (IRT) approach including the score of specific items from the three applied rating scales, i.e. the Montgomery-Åsberg Depression Rating Scale, Hamilton Rating Scale for Depression and the Beck Depression Inventory. Weeks $9-12$ before switching are not shown because of too few individuals.

antidepressant treatment effects. Future large trials need to include patients with MDD and non-response after antidepressant treatment of sufficient length and dose and compare: (a) different randomised intervention groups (for example continuation/up-titration, augmentation or switching), (b) include several antidepressant drugs with different receptor profiles, and (c) explore the importance of specific timing of the new intervention (for example early versus late switching).

Ole Köhler-Forsberg (ID, MD, PhD-Student, Psychosis Research Unit, Aarhus University Hospital - Psychiatry; Department of Clinical Medicine, Aarhus University; and iPSYCH, The Lundbeck Foundation Initiative for Integrative Psychiatric Research, Denmark; Erik Roj Larsen, MD, PhD, Chief Physician, Department of Psychiatry,

Psychiatry in the Region of Southern Denmark, Institute of Clinical Research, Research Unit of Psychiatry, University of Southern Denmark, Denmark; Henriette N. Buttenschøn, MSC, PhD, Associate Professor, IPSYCH, The Lundbeck Foundation Initiative for Integrative Psychiatric Research; and Translational Neuropsychiatry Unit, Department of Clinical Medicine, Aarhus University, Denmark; Marcella Rietschel, MD, PhD, Professor, Central Institute of Mental Health, Department of Genetic Epidemiology in Psychiatry, Medical Faculty Mannheim/Heidelberg University, Germany; Joanna Hauser, MD, PhD, Professor, Laboratory of Psychiatric Genetics, Department of Psychiatry, Poznan University of Medical Sciences, Poland; Daniel Souery, MD, PhD, Professor, Laboratoire de Psychologie Médicale, Université Libre de Bruxelles; Psy Pluriel - Centre Européen de Psychologie Médicale, Belgium; Wolfgang Maier, MD, PhD Professor, Department of Psychiatry, University of Bonn, Germany; Anne Farmer, MD, PhD, Professor, Social, Genetic and Developmental Psychiatry Centre, Institute of Psychiatry, Psychology and Neuroscience, King's College London, UK; Peter McGuffin, MD, PhD, Professor, Social, Genetic and Developmental Psychiatry Centre, Institute of Psychiatry, Psychology and Neuroscience, King's College London, UK; Katherine J. Aitchison, MD, PhD, Professor, University of Alberta, Canada; Rudolf Uher (ID, MD, PhD, Professor, Social, Genetic and Developmental Psychiatry Centre, Institute of Psychiatry, Psychology and Neuroscience, King's College London, UK; and Department of Psychiatry, Dalhousie University, Canada; Ole Mors, MD, PhD, Professor, Psychosis Research Unit, Aarhus University Hospital - Psychiatry; Department of Clinical Medicine, Aarhus University; and iPSYCH, The Lundbeck Foundation Initiative for Integrative Psychiatric Research, Denmark

Correspondence: Ole Köhler-Forsberg, Psychosis Research Unit, Aarhus University Hospital, Risskov, Skovagervej 2, DK-8240 Risskov, Denmark. Email: karkoe@rm.dk

First received 29 Sep 2018, final revision 14 Nov 2018, accepted 11 Dec 2018 


\section{Funding}

The Genome-Based Therapeutic Drugs for Depression (GENDEP) study was funded by a European Commission Framework six grant (EC Contract Ref LSHB-CT-2003-503428). Lundbeck provided both nortriptyline and escitalopram free of charge for the GENDEP study. The funders had no role in the design and conduct of the study, in data collection, analysis, or interpretation or in writing the report.

\section{Supplementary material}

Supplementary material is available online at https://doi.org/10.1192/bjp.2018.302.

\section{References}

1 Bayes AJ, Parker GB. Comparison of guidelines for the treatment of unipolar depression: a focus on pharmacotherapy and neurostimulation. Acta Psychiatr Scand 2018; 137: 459-71.

2 Cipriani A, Furukawa TA, Salanti G, Chaimani A, Atkinson LZ, Ogawa Y, et al. Comparative efficacy and acceptability of 21 antidepressant drugs for the acute treatment of adults with major depressive disorder: a systematic review and network meta-analysis. Lancet 2018; 391: 1357-66.

3 Pereira VS, Hiroaki-Sato VA. A brief history of antidepressant drug development: from tricyclics to beyond ketamine. Acta Neuropsychiatr 2018; 30: 307-22

4 Trivedi MH, Rush AJ, Wisniewski SR, Nierenberg AA, Warden D, Ritz L, et al. Evaluation of outcomes with citalopram for depression using measurementbased care in STAR*D: implications for clinical practice. Am J Psychiatry 2006; 163: $28-40$

5 Bose A, Tsai J, Li D. Early non-response in patients with severe depression: escitalopram up-titration versus switch to duloxetine. Clin Drug Investig 2012: 32: $373-85$

6 Nierenberg AA, Fava M, Trivedi MH, Wisniewski SR, Thase ME, McGrath PJ, et al. A comparison of lithium and T(3) augmentation following two failed medication treatments for depression: a STAR ${ }^{D}$ report. Am J Psychiatry 2006; 163: 1519-30

7 Thase ME, Rush AJ, Howland RH, Kornstein SG, Kocsis JH, Gelenberg AJ, et al Double-blind switch study of imipramine or sertraline treatment of antidepressant-resistant chronic depression. Arch Gen Psychiatry 2002; 59: 233-9.

8 Rush AJ, Trivedi MH, Wisniewski SR, Stewart JW, Nierenberg AA, Thase ME, et al. Bupropion-SR, sertraline, or venlafaxine-XR after failure of SSRIS for depression. N Engl J Med 2006; 354: 1231-42.

9 Bschor T, Baethge C. No evidence for switching the antidepressant: systematic review and meta-analysis of RCTs of a common therapeutic strategy. Acta Psychiatr Scand 2010; 121: 174-9.

10 Bschor T, Kern H, Henssler J, Baethge C. Switching the antidepressant after nonresponse in adults with major depression: a systematic literature search and meta-analysis. J Clin Psychiatry 2018; 79: 10.

11 Souery D, Serretti A, Calati R, Oswald P, Massat I, Konstantinidis A, et al. Citalopram versus desipramine in treatment resistant depression: effect of continuation or switching strategies: a randomized open study. World J Biol Psychiatry 2011; 12: 364-75.

12 Mohamed S, Johnson GR, Chen P, Hicks PB, Davis LL, Yoon J, et al. Effect of antidepressant switching $v$. augmentation on remission among patients with major depressive disorder unresponsive to antidepressant treatment: the VAST-D randomized clinical trial. JAMA 2017; 318: 132-45

13 Brignone M, Diamand F, Painchault C, Takyar S. Efficacy and tolerability of switching therapy to vortioxetine versus other antidepressants in patients with major depressive disorder. Curr Med Res Opin 2016; 32: 351-66.

14 Shelton RC, Williamson DJ, Corya SA, Sanger TM, Van Campen LE, Case M, et al. Olanzapine/fluoxetine combination for treatment-resistant depression: a controlled study of SSRI and nortriptyline resistance. J Clin PSychiatry 2005; 66 : 1289-97.

15 Connolly KR, Thase ME. If at first you don't succeed: a review of the evidence for antidepressant augmentation, combination and switching strategies. Drugs 2011; 71: 43-64.

16 Nakajima S, Uchida H, Suzuki T, Watanabe K, Hirano J, Yagihashi T, et al. Is switching antidepressants following early nonresponse more beneficial in acute-phase treatment of depression?: a randomized open-label trial. Prog Neuropsychopharmacol Biol Psychiatry 2011; 35: 1983-9.

17 Uher R, Farmer A, Maier W, Rietschel M, Hauser J, Marusic A, et al. Measuring depression: comparison and integration of three scales in the GENDEP study. Psychol Med 2008; 38: 289-300.

18 Wing J, Sartorius N, Üstun T. WHO Diagnosis and Clinical Measurement in Psychiatry. A Reference Manual for SCAN. Cambridge University Press, 1998.

19 Uher R, Maier W, Hauser J, Marusic A, Schmael C, Mors O, et al. Differential efficacy of escitalopram and nortriptyline on dimensional measures of depression. Br J Psychiatry 2009; 194: 252-9.

20 Lingjaerde O, Ahlfors UG, Bech P, Dencker SJ, Elgen K. The UKU side effect rating scale. A new comprehensive rating scale for psychotropic drugs and a crosssectional study of side effects in neuroleptic-treated patients. Acta Psychiatr Scand 1987; Suppl 334: 1-100.

21 Montgomery SA, Åsberg M. A new depression scale designed to be sensitive to change. Br J Psychiatry 1979; 134: 382-9.

22 Hamilton M. A rating scale for depression. J Neurol Neurosurg Psychiatry 1960; 23: $56-62$

23 Beck AT, Ward CH, Mendelson M, Mock J, Erbaugh J. An inventory for measuring depression. Arch Gen Psychiatry 1961; 4: 561-71.

24 Lane P. Handling drop-out in longitudinal clinical trials: a comparison of the LOCF and MMRM approaches. Pharm Stat 2008; 7: 93-106.

25 Papakostas GI, Fava M, Thase ME. Treatment of SSRI-resistant depression: a meta-analysis comparing within- versus across-class switches. Biol Psychiatry 2008; 63: 699-704

26 Uher R, Mors O, Rietschel M, Rajewska-Rager A, Petrovic A, Zobel A, et al. Early and delayed onset of response to antidepressants in individual trajectories of change during treatment of major depression: a secondary analysis of data from the genome-based therapeutic drugs for depression (GENDEP) study. J Clin Psychiatry 2011; 72: 1478-84 